Upcoming: October 3-5 Three-Dimensional Multifunctional Ceramic Composites Workshop, MRS.

To list an event in the Calendar, contact J. Meiksin, Materials Research Society, 506 Keystone Drive, Warrendale, PA 150867573; 724-779-3004 ext. 522 fax 724-779-8313; bulletin@mrs.org.

MRS, A-MRS, B-MRS, C-MRS, E-MRS, MRS-A, MRS-I, MRSJ, MRS-K, MRS-R, MRS-S, MRS-T, M-MRS, or IUMRS at the end of an entry indicates sponsorship or co-sponsorship of an event by the International Union of Materials Research Societies or one of its adhering bodies. "Endorsed" identifies events endorsed by MRS.

$\nabla$ identifies a new or revised entry this month.

See the July 2005 MRS BULLETIN for September 2005 Calendar entries. For updates, access www.mrs.org/ meetings/other/.

\section{OCTOBER 2005}

2-5 3rd Fracture and Flow in Advanced Glasses Conf., University Park, PA. Carlo Pantano; ars2@psu.edu.

2-5 Powder Metallurgy Congress and Exhibition (PM2005) Prague, Czech Republic. Powder Metallurgy, Old Bank Buildings, Bellstone, Shrewsbury, United Kingdom, 44-0-1743-248899; fax 44-0-1743-362968; e-mail info@ epma.com; www.epma. com/pm2005

\section{3-5 Three-Dimensional Multifunctional Ceramic} Composites Workshop, Urbana, IL. Materials Research Society, 506 Keystone Drive, Pittsburgh, PA 15086-7573; 724-779-3003; fax 724-779-8313; e-mail info@mrs.org; www.mrs.org/ meetings/workshop. MRS

3-5 $\boldsymbol{\nabla}$ Intl. Seminar: Commercial Applications for Inherently Conductive Polymers, Boston, MA. Matt Aldissi, Advanced Polymer Courses, 200 9th Avenue North, Suite 100, Safety Harbor, FL 34695; 727-723-3006; fax 727-723-3007; e-mail maldissi@fractalsystemsinc. com; www.conductivepolymers. com/semcont.htm

4-6 2nd Intl. Symp. on Point Defect and Nonstoichiometry ISPN-2, Kaohsiung, Taiwan. National Sun Yat-Sen University, Mitch Chou, Kaohsiung 804 Taiwan, 886-7-5252000 ext; fax 886-7-5254099; e-mail mitch@ mail.nsysu.edu.tw; www.mse. nsysu.edu.tw/ispn2005.
4-6 Grove Fuel Cell Symp., London, United Kingdom. Gill Heaton, Elsevier, Hillside Cottages, Wheatley Rd., Islip, Oxford, United Kingdom, 44-1865-373625; fax 44-1865-375855; e-mail wom-conference@elsevier.com; www.grovefuelcell.com.

\section{4-8 $\nabla$ European Conf. on} Chemistry for Life Sciences, Rimini, Italy. Fax 39-0547-382348; e-mail lifescience@unibo.it; www.lifescience-fecs.org.

5-6 Microsystem

Technologies 2005, Munich, Germany. www.mesago.com/mst.

6-7 IACC—Intl. Activated Carbon Conf., Pittsburgh, PA. Henry Nowicki, PACS Testing and Consulting, 409 Meade Drive, Coraopolis, PA, 15108; 724-4576576; fax 724-457-1214;

e-mail hnpacs@aol.com; www. pacslabs.com.

9-14 16th Conf. on Enzyme Engineering, Gyeong-ju, South Korea. ECI, 6 Metro Tech Center, Brooklyn, NY, 11201; 718-2603743; fax 718-260-3754; e-mail info@eci.poly.edu; www.engconfintl.org/5ae.html

9-14 ECl-Instrumented Indentation Testing in Materials Research and Development, Crete, Greece. E-mail info@eci.poly.edu; www.engconfintl.org/5av.html.

12-16 AIOM-Advances in Optical Materials, Tucson, AZ. Sophie Peters AIOM Conference Secretariat, Elsevier, The Boulevard, Langford Lane, Kidlington, Oxford 0X5 1GB, United Kingdom, 44-01865-843643; fax 44-0-1865843958; e-mail s.peters@elsevier. com; www.aiom.elsevier.com.

14-19 $\nabla$ ESF-Supramolecula Chemistry, Obernai, France. European Science Foundation, ESF Research Conferences, 1, quai Lezay Marnésia, BP 90015 , Strasbourg Cedex, France, 33-0388-76-71-00; www.esf.org.

16-20 OSA Frontiers in Optics, Tucson, AZ. Optical Society of America, 2010 Massachusetts Ave., NW, Washington, 202-2238130; fax 202-223-1096; e-mail info@osa.org; www. frontiersinoptics.org.

16-21 ECS-Intl. Symp. on High Dielectric Constant Gate Stacks, Los Angeles, CA. The Electrochemical Society,

65 S. Main Street, Building D, Pennington, NJ, 08534-2839; 609-737-1092; fax 609-737-2743; e-mail ecs@electrochem.org; www.electrochem.org.
17-19 Light Emitting Diodes 2005, San Diego, CA. Intertech USA, INTERTECH, 19 Northbrook Drive, Portland, ME, 04105; 207-781-9800; fax 207-781-2150; e-mail info@Intertechusa.com; www.intertechusa.com/leds.html.

17-20 IEEE Integrated Reliability Workshop 2005, Fallen Leaf Lake CA. IRW, P.0. Box 308;

Westmoreland, NY 13490-0308;

315-339-3968, fax to 315-336-

9134, becky@sar101.com; www.iirw.org.

17-21 ASNT Fall Conf. and Quality Testing Show 2005, Columbus, $\mathrm{OH}$. Kelly Thomas, American Society for Nondestructive Testing, P.O. Box 28518, 1711 Arlingate $\mathrm{Ln}$, Columbus, $\mathrm{OH} 43228$ 0518; 800-222-2768 x227; fax 614274-6899; kthomas@asnt.org; www.asnt.org.

17-21 Intl. Astronautica Congress (IAC) 2005, Fukuoka, Japan. Secretariat Intl. Astronautical Congress 2005, 1-18-2 Shinbashi Minato-ku, Tokyo, Japan, 81-33503-8151; fax 81-3-3503-8152; e-mail info@iac2005.org;

www.iac2005.org.

29-29 $\nabla$ Annual Dartmouth Nanomaterials Symposium, Hanover, NH. Dartmouth College, Center for Nanomaterials Research at Dartmouth, Thayer School of Engineering, 8000 Cummings Hall, Hanover, NH 03755; 603-6462184; fax 603- 646-3856; e-mail nanomaterials@dartmouth.edu thayer.dartmouth.edu/other/ nanomaterials/symposium.shtml.

30-3 SAMPE-Intl. Fal Technical Conf. (ISTC), Seattle, WA. SAMPE, e-mail registration@ sampe.org; www.sampe.org.

30-4 AVS Intl. Symposium, Boston, MA. AVS, 110 Yellowstone Drive, Suite 120, Chico, CA 95973; 530-896-0477; fax 530896-0487; e-mail della@avs.org; www.avs.org.

31-2 V World Conf. on Physics for Sustainable Development (WCPSD), Durban, South Africa. www.wcpsd.org.

31-3 ICALEO - IntI. Congress on Applications of Lasers and Electro-Optics, Miami, FL. Laser Institute of America, 13501 Ingenuity 407-380-1553; fax 407-380-5588; e-mail conferences@laserinstitute. org; www.icaleo.org.

\section{NOVEMBER 2005}

1-3 ICNT-Intl. Congress of Nanotechnology 2005, San Francisco, CA. ICNT, IANANO, Inc. 2386 Fair Oaks Blvd., Sacramento, CA, 95825; e-mail info@ianano.org; www.nanotechcongress.com. Drive, Suite 128, Orlando, FL, 32826;
5-11 ASME Intl. Mechanical Engineering Congress and Exposition, Orlando, FL. ASME Kim Punter, 212-591-8258; e-mail punterk@asme.org; www.asme.org.

7-10 Instituto Argento de Siderurgia (IAS) Steelmaking

Conference \& Ironmaking

Conference, San Nicolas, Argentina Cristian Genzano, Instituto Argentino de Siderur, Av. Central y Calle 19 Oeste, 2900 San Nicolas, Buenos Aires, Argentina, 54-3461460803; fax 54-3461-462989; e-mail genzano@siderurgia.org.ar; www.siderurgia.org.ar/conferencia Call_for_papers.htm.

8-10 $\nabla$ Aerospace Testing Expo, Long Beach, CA. Aerospace Testing Expo (N. Am.), 44-0-1306743744; e-mail ianstone@ ukintpress.com; www. aerospacetesting-expo. com/northamerica.

8-11 $\nabla$ UNITECR Conf. for Refractory Technology, Orlando, FL. The American Ceramic Society, American Ceramic Society, Westerville, $\mathrm{OH}, 43081$;

e-mail info@ceramics.org; www.unitecr.org.

13-17 SETAC North America Annual Meeting, Baltimore, MD.

SETAC North America, 1010 North 12th Avenue, Pensacola, FL, 32501 3370; 850-469-1500; fax 580-469 9778; e-mail setac@setac.org; www.setac.org.

14-16 Continuous Casting of Non-Ferrous Metals Intl. Conf. \& Exhibition, Neu-UIm, Germany. Vera Hausen, Niels Parusel, DGM Secretariat, Deutsche Gesellschaft für Materialkunde, Hamburger Alle 26, Frankfurt, Germany, 49-697917-747; fax 49-69-7917-733; e-mail concast@dgm.de; www.dgm.de/concast.

15-18 $\nabla$ Productronica 2005, Munich, Germany. E-mail info@productronica.de; www.productronica.de.

22-25 Ninth NCB Intl. Seminar on Cement, New Delhi, India. NCCBM, Ninth NCB, 34 Km Stone, Delhi Mathura Road (NH2), Ballabgarh 121 004, Haryana India, 91-129-2242051-56; fax 91-129-2242100, 2246175 e-mail nccbm@giasd101.vsnl.net.in.

\section{8-2 MRS Fall Meeting,}

Boston, MA. Materials Research Society, 506 Keystone Drive, Warrendale, PA 15086-7573 724-779-3003; fax 724-779-8313 e-mail info@mrs.org; www.mrs.org. MRS.

\title{
DECEMBER 2005
}

2-3 European Coatings Conf. The Power of Ink Jet Materials, Berlin, Germany. Vincentz Network, Amanda Beyer, Schiffgraben 43 30175 Hannover, Germany, 49-511 99-10-271; fax 49-511-99-10-279; e-mail amanda.beyer@coatings.de; 
23-26 Thermal Solutions Sarasota, FL. Snell InfraredThermal Solutions, 100 State Street, Suite 200, Montpelier, VT, 05602; 800-636-9820; e-mail info@snellinfrared.com; www. thermalsolutions.org.

\section{FEBRUARY 2006}

15-17 $\nabla$ Smart Coatings 2006 Orlando, FL. Coatings Research Institute, Sandra Tanner, 430 W. Forest Ave., Ypsilanti, MI, 48197; 734-487-2203; fax 734-483-0085; e-mail sandy.tanner@emich.edu; www.emich.edu/public/ coatings_research/smartcoatings/ index2.html.

19-24 $\nabla$ SPIE-Microlithography Conference, San Jose, CA. SPIE Meetings, P.0. Box 10, Bellingham, WA, 98227-0010; 360-676-3290; fax 360-647-1445; e-mail spie@ spie.org; spie.org/conferences.

27-1 Intl. Conf. on Superhard Coatings, Ein-Gedi, Israel. E-mail hardcoat@eng.tau.ac.il;

www.hardcoat.org.

\section{MARCH 2006}

12-16 2006 TMS Annual Meeting and Exhibition, San Antonio, TX

TMS Meetings Services, 184 Thorn Hill Road, Warrendale, PA, 15086; 724-776-9000 x243; fax 724-7763770; e-mail mtgserv@tms.org www.tms.org/meetings/meetings.asp.

13-17 2006 APS March Meeting Baltimore, MD. American Physical Society, e-mail meetings@aps.org www.aps.org/meet.

17-18 SPE-European Thermoforming Conf., Salzburg, Austria. SPE-Europe, Belgium, 32-0-3-541-77-55; fax 32-0-3-541 84-25; e-mail spe.europe@pi.be; www.4spe.org/conf/

0603eutherm.php.

\section{APRIL 2006}

17-21 2006 MRS Spring Meeting, San Francisco, CA Materials Research Society, 506 Keystone Drive, Warrendale, PA 15086; 724-779-3003; fax 724779-8313; e-mail info@mrs.org; www.mrs.org. MRS.

22-27 49th SVC Annua Technical Conf., Washington, D.C. Society of Vacuum Coaters, 505856-7188; e-mail svcinfo@svc.org www.svc.org.

27-30 SFB 2006-Society for Biomaterials Annual Meeting and Exposition, Pittsburgh, PA Rebecca Hanes Society for Biomaterials, 17000 Commerce Parkway, Suite, Mt. Laurel, 856439-0826 x3061; fax 856-4390525; e-mail rhaines@ahint.com www. biomaterials.org/Meetings/ meetings.htm.

\section{MAY 2006}

7-11 $\nabla$ SPE-Annual Technical Conference (ANTEC), Charlotte, NC. Society of Plastics Engineers SPE-Europe, Belgium, 32-0-3541-77-55; fax 32-0-3-541-84-25; e-mail spe.europe@pi.be; www.speeurope.org.

15-17 Intl. Thermal Spray Conf. and Exposition, Seattle, WA International ASM, 9639 Kinsman Rd., Materials Park, 800-336-5152 ext 6; fax 440-338-4634; e-mail Cust-srv@asminternational.org; www.asminternational.org/itsc.

22-26 ICMOVPE XIII-Int. Conf. on Metal-Organic Vapor Phase Epitaxy, Miyazaki, Japan. Yoshinao Kumagai, Tokyo University of A \& T, Koganei, Tokyo 184-8588, Japan, 81-42-388-7469; fax 81-42386-3003; e-mail icmovpe@cctuat. ac.jp; icmovpe13.chem.tuat.ac.jp
28-2 $\nabla$ MCWASP-Modeling of Casting, Welding and Advanced Solidification Processes Conference, Opio, France. ESA Conference Bureau/Congrex Holland BV, MCWASP, P.0. Box 299, AG Noordwijk, Netherlands, 31-71565-5005; fax 31-71-565-5658; e-mail Esa.Conference.Bureau@ esa.int; www.mcwasp.org.

\section{JUNE 2006}

6-8 Magneto-0ptica Recording Intl. Symp. 2006 (MORIS 2006) Workshop, Chiba Prefecture, Japan. MORIS 2006 Workshop Secretariat, Proactive Inc., Promena Kobe 16F, 1-8-1, Higashikawasaki-cho, Chuo-ku, Kobe 650-0044, Japan; 81-78366-5050; fax 81-78-366-5051 moris2006ws@pac.ne.jp; www.ecs.cst.nihon-u.ac.jp/ moris2006ws. Endorsed.

25-29 Intl. Congress on Ceramics, Toronto, Canada. American Chemical Society, 1155 Sixteenth Street NW, Washington, DC, 20036; (202) 872-4374; fax (202) 872-6128; e-mail natlmtgs@acs.org; www.ceramics.org.

\section{JULY 2006}

2-7 21st International Liquid Crystal Conference, Keystone, $\mathrm{CO}$. Conference Management Office, Kinsley \& Associates, LLC, 303.798 3664; ilcc@kinsleyassociates.com; wWW.ilcc2006.com.

\section{AUGUST 2006}

6-10 $\nabla$ Microscopy and Microanalysis 2006, Chicago, IL. Microscopy/Marketing \& Education, Inc., Meeting Management Microscopy \& Microanalysis, 125 Parido Street, Suite 102 Springfield, MA, 01118-2130 972-954-8011; fax 972-954-8018; e-mail corporate@mme1.com; microscopy.org/MMMeetings.
20-26 Intl. Conf. on Nanostructured Materials, Bangalore, India. Prof. K. Chattopadhyay, Dept. of Metallurgy, Indian Institute of Science, Bangalore 560012 India; 91-80-22932262; fax 91-80 23601991; e-mail nano2006@met. iisc.ernet.in; met.iisc.ernet.in/ nano2006.

27-31 TMS-Sohn Intl. Symp. on Advanced Materials:

Principles, Technologies, and Industrial Practice, San Diego, CA. TMS Meetings Services, 184 Thor Hill Road, Warrendale, PA, 15086 ; 724-776-9000 x243; fax 724-776 3770; e-mail mtgserv@tms.org; www.tms.org/Meetings/Specialty/ FallExt2006/FallExt2006-Home. $\mathrm{html}$. Endorsed.

\section{SEPTEMBER 2006}

10-15 ESOMAT-European Symp. on Martensitic Transformations, Bochum, Germany. ESOMAT, e-mail esomat06@ruhr-uni-bochum. de; www.ruhr-uni-bochum. de/esomat06.

24-28 $\boldsymbol{\nabla}$ Intl. Symp. on Crystallization in Glasses and Liquids, Jackson Hole, WY. The American Ceramic Society, 735 Ceramic Place, Suite 100 Westerville, OH, 43081; 614-8904700; fax 614-899-6109; e-mail info@ceramics.org; www.ceramics. org/meetings/crystallization.

\section{OCTOBER 2006}

\section{3-6 $\boldsymbol{\nabla}$ World Congress on} Emulsions, Lyon, France 140 cours Charlemagne 69002 Lyon, France; 33-0-4-72-77-45-50; fax 33-0-4-72-77-45-77; e-mail cme@ package.fr; www.cme-emulsion.com.

15-18 $\nabla$ Material Science and Technology Conf. and Exhibition (MS\&T06), Cincinnati, OH. The American Ceramic Society, 735 Ceramic Place, Suite 100

Westerville, OH, 43081; 614-890-

4700; fax 614-899-6109;

e-mail info@ceramics.org;

www.ceramics.org/meetings.

\section{NOVEMBER 2006}

27-1 2006 MRS Fall Meeting Boston, MA. Materials Research Society, 506 Keystone Drive, Warrendale, PA 15086; 724-7793003; fax 724-779-8313; e-mail info@mrs. org; www.mrs.org. MRS

\section{FEBRUARY 2007}

20-23 $\nabla$ Intl. Conf. on Recent Advances in Composite Materials (ICRACM), New Delhi, India.

V.K. Srivastava, ICRACM-2007, Dept. of Mechanical Engineering Institute of Technology, Banaras Hindu University, Varanasi221005, India; 91-542-2368157; e-mail vijays_sa@yahoo.com; www.htu.ac.in/notification/ icracm.htm.

25-1 2007 TMS Annual Meeting and Exhibition, Orlando, FL. TMS Meetings Services, 184 Thorn Hill Road, Warrendale, PA 15086; 724-776-9000 x243; fax 724-776-3770; e-mail mtgserv@ tms.org; www.tms.org/meetings/ meetings.asp.

\section{APRIL 2007}

\section{9-13 2007 MRS Spring} Meeting, San Francisco, CA. Materials Research Society, 506 Keystone Drive, Warrendale 724-779-3003; fax 724-779-8313 e-mailinfo@mrs.org; www.mrs.org. MRS

\section{AUGUST 2007}

6-9 $\nabla$ Microscopy and Microanalysis 2007, Ft Lauderdale, FL. Microscopy/Marketing \& Education, Inc., Meeting Management Microscopy \& Microanalysis, 125 Parido Street, Suite 102, Springfield, MA 01118-2130; 972-954-8011; fax 972-954-8018; e-mail corporate@ mme1.com; microscopy.org/ MMMeetings.

\section{Mis Entrepreneurship Ghallonge}

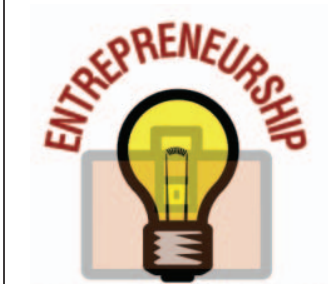

CHALLENGE
Got an Idea? Interested in bringing a new technology to the marketplace?

Check out the MRS Entrepreneurship Challenge!

- $\$ 30001$ st prize

- \$3000 travel allowance and complimentary registration for semi-finalists to attend the MRS 2006 Spring Meeting in San Francisco, CA

- Develop the entrepreneurial skills needed to get your technology out of the lab and into the marketplace

For more information, go to www.mrs.org/entrepreneur/ 